\title{
Harmonic emission study of individual wind turbines and a wind park
}

\author{
K. Yang ${ }^{1}$, S. Cundeva ${ }^{2}$, M. Bollen ${ }^{1}$ and M. Wahlberg ${ }^{1}$ \\ ${ }^{1}$ Luleå University of Technology \\ Department of Engineering Sciences and Mathematics \\ Skellefteå, Sweden \\ e-mail: kai.yang@ltu.se, math.bollen@ ltu.se, mats.wahlberg@ ltu.se \\ ${ }^{2}$ University Ss Cyril and Methodius \\ Faculty of Electrical Engineering and Information Technologies, Skopje, Macedonia \\ e-mail: scundeva@feit.ukim.edu.mk
}

\begin{abstract}
The paper analyses the measured emission from four individual modern turbines of about 2MW size equipped with power electronics. The four turbines show different long term spectra and their spectra also show different variations with time. The harmonic emission from individual turbines consists of harmonic and interharmonic spectra. This emission was shown to be low, with the exception of certain non-characteristic frequency components. The measured emission from individual wind turbines has been compared with emission limits. When IEEE Std 519 is applied, for two of the turbines the limits are exceeded for higher odd harmonics. At the same time, these turbines comply with the national French emission limits. Next, a study of the primary harmonic emission of a wind park as a whole was performed. It is concluded that the emission from a park can be higher as well as lower than the emission that would be obtained by adding the harmonic magnitudes of the individual turbine emissions.
\end{abstract}

Key words: power quality, wind power, harmonic distortion, harmonic spectra

\section{Introduction}

Modern, MW-size, wind turbines contain power-electronic converters. This can either be a full-power converter or a part-size converter in a double-fed induction generator. The presence of power-electronic converters makes that distorted currents are expected and that there is concern from network operators that distortion levels in the grid will increase with increased penetration of windpower. Harmonic studies are therefore a common requirement as part of the connection agreements for individual turbines as well as wind parks. The subject is generally forgotten in general discussions about the impact of wind power on the grid; on the other hand is the harmonic emission from wind power mentioned as a serious concern by a small group of experts. Both are a serious oversimplification: harmonic emission should not be forgotten, but in most cases there is no reason for concern.
This paper covers the harmonic emission from individual wind turbines (to be discussed in Section 2) and from a wind park as a whole (the subject of Section 3).

\section{Harmonic emission from individual turbines}

Measurements have been performed of the emission from four turbines of 1.8 to $2.5 \mathrm{MW}$ size, equipped with power-electronics [1]. For example, the first turbine Nordex 90 is a type-3 wind turbine with a rated power $2.5 \mathrm{MW}$. It is equipped with a gearbox of multi-stage planetary plus one stage spur gear, a six-pole doubly-fed asynchronous generator (DFIG). A partial rated converter is installed between the rotor and line connection. It is designed as a DC voltage link converter with IGBT technology. The rotor outputs a pulse-width modulation (PWM) modulated voltage, while the stator outputs $3^{*} 660 \mathrm{~V} / 50 \mathrm{~Hz}$ voltage. The wind turbine is connected to the collection grid through one medium-voltage (MV) transformer, which is housed in a separate transformer station beside the turbine foundation.

The other 3 turbines are Vestas V90 - type-3 wind turbine with a rated power of 2 MW, Enercon E82 - type- 4 wind turbine with a rated power of $2 \mathrm{MW}$ and Enercon E 40 with rated power of $3 \times 600 \mathrm{~kW}$.

\section{A. Long term spectrograms}

The variation of the emission of the turbines with time is shown in Figure 1 as a time-frequency chart: a so-called "long-term spectrogram". The horizontal scale gives the time in days; the vertical scale the frequency (in multiples of the $50 \mathrm{~Hz}$ fundamental frequency) and the intensity of the emission is given in the form of a color intensity scale: blue is lowest intensity; red is highest intensity. The vertical dark blue parts are idle state of the wind turbine. 

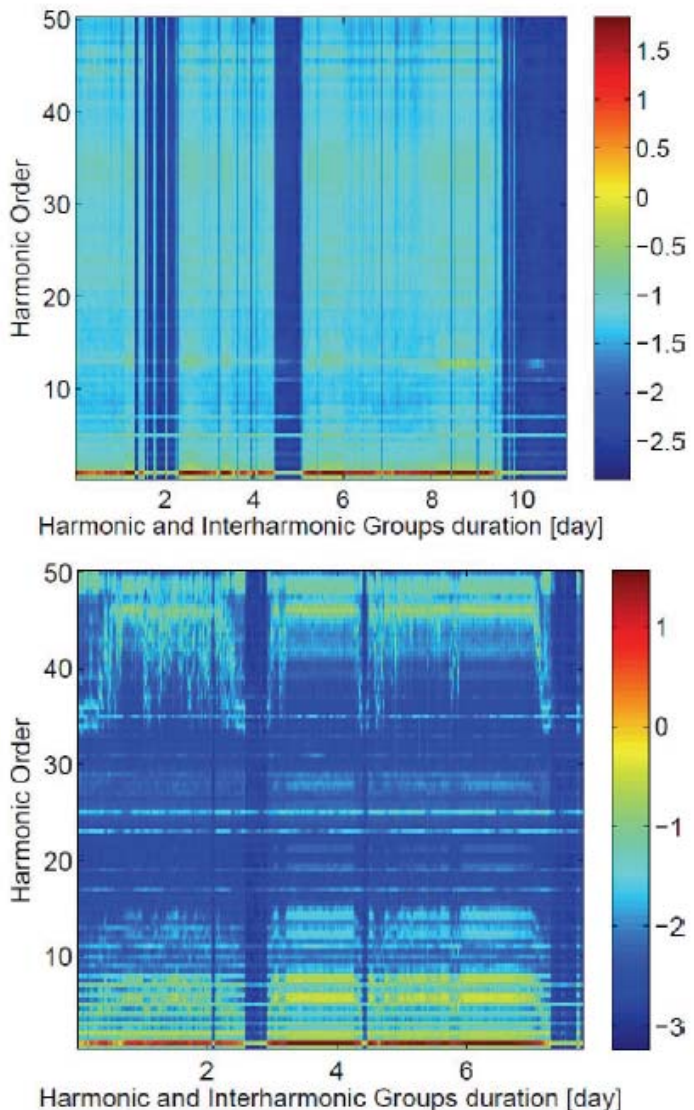
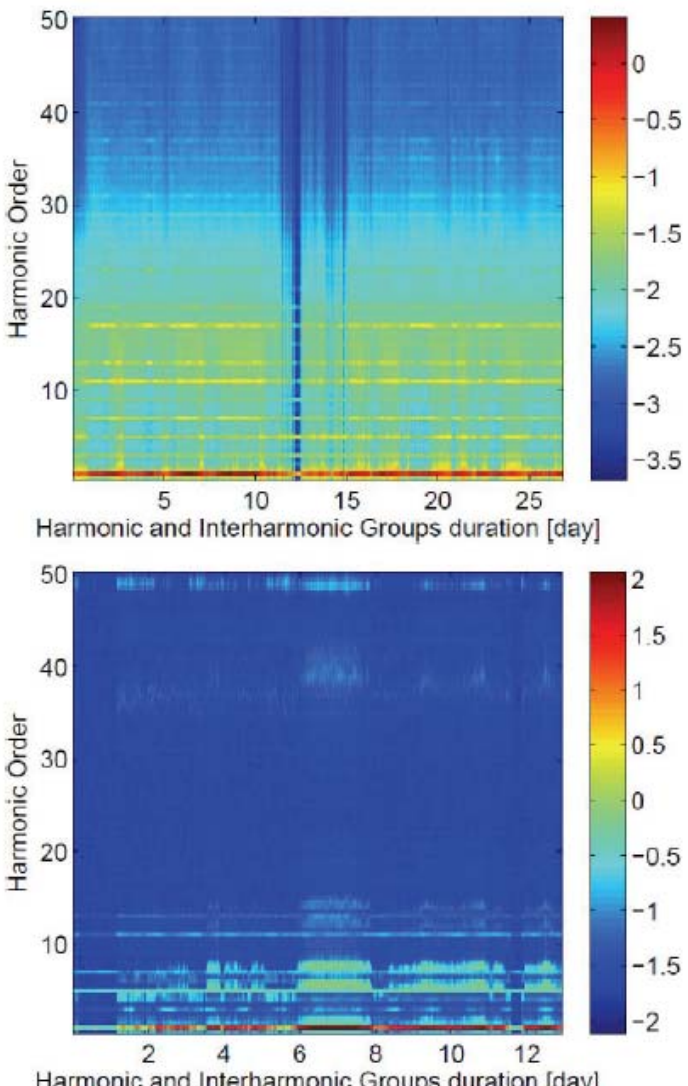

Harmonic and Interharmonic Groups duration [day]

Fig.1. Variation in time of the emission from four modern wind turbines.

The four turbines not only show different spectra; their spectra also show different variations with time. For example, the upper left plot shows that the turbine emits a continuous spectrum (light blue) most of the time, but that during certain periods (the dark blue vertical bands) this continuous spectrum disappears and only a small number of frequency components (harmonics 3, 5, 7, 11 and 13) remain. From the magnitude of the $50-\mathrm{Hz}$ component (the thick line at the bottom of the plot, red most of the time) we see that this occurs when the emission from the turbine is low.

The bottom right figure shows that the emission is small between harmonic 15 and 35. During days 6 and 7, emission occurs are frequencies that are not there on other days. The $50-\mathrm{Hz}$ line gets dark red during these days, which indicates high amount of wind-power production.

Further studies have shown that the variation of the spectra with time is not only due to the variation of produced power with time.

\section{B. Harmonic spectra}

The emission spectrum of the three largest turbines is shown in Fig. 2. The measurements were performed on the medium-voltage side of the turbine transformer during a period between 8 and 13 days. The harmonic and interharmonics subgroups, as defined in IEC 61000-4-7, were obtained every 10 minutes. The figure shows 95percentile of the harmonic subgroups as a percentage of the 95-percentile of the fundamental component (the latter was close to the rated current). The highest of the values for the three phases was chosen.

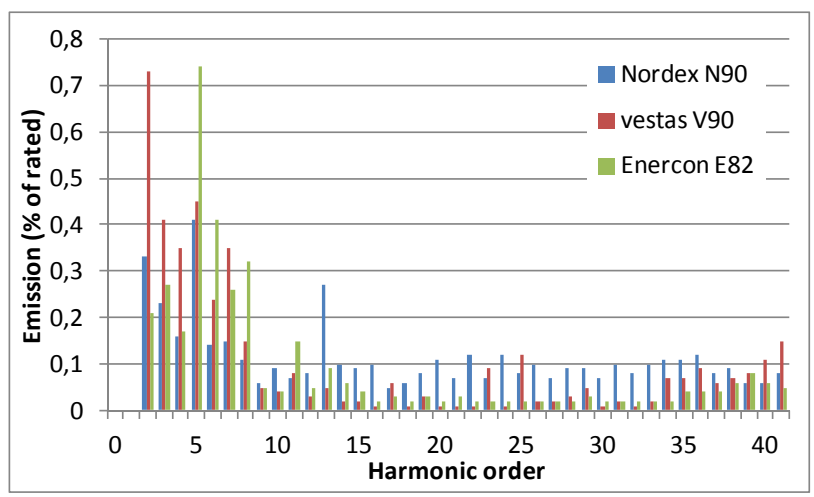

Fig. 2. Emission spectrum from three modern MWclass wind turbines.

There are differences as well as similarities between the three spectra. All three turbines show their predominant emission up to about order 10; they also all three show increased emission between harmonic 35 and 40 .

\section{Interharmonic spectra}

Interharmonic frequency is any frequency which is a non-integer multiple of the fundamental frequency. For comparison, the interharmonic spectra of the wind turbines, obtained in the same way, are shown in Fig. 3. The emission at interharmonic frequencies is similar in magnitude to the emission at harmonic frequencies. The spectra of the three turbines are different also for 
interharmonics, but a general observation is that all of them emit distortion at interharmonic frequencies.

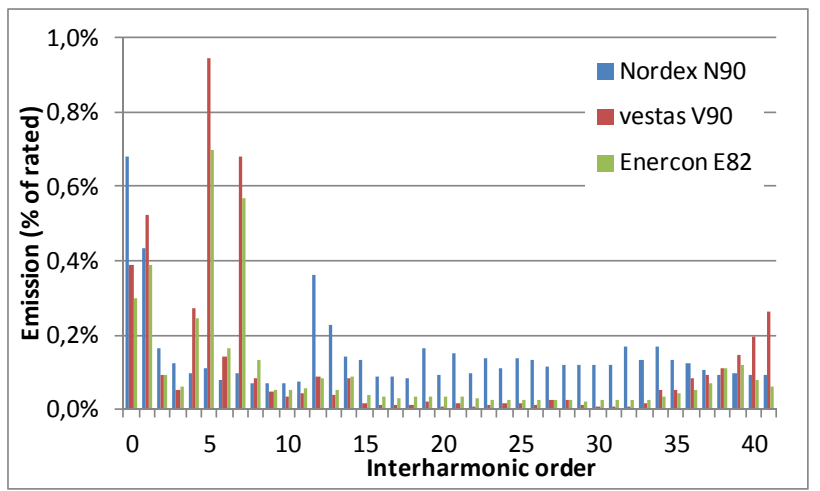

Fig. 3. Emission spectrum from three modern MV-class wind turbines.

\section{Emission limits}

Excessive amounts of harmonic emission from wind-power installations (or from any other installation) impact the grid in two different ways:

$\checkmark$ Excessive harmonic currents through grid components like transformers;

$\checkmark$ Excessive harmonic voltage distortion for other network users.

For example, excessive harmonic currents cause increased heating of components in the grid (especially transformers are sensitive to this) resulting in a reduced lifetime of these components.

Limits on harmonic voltage emission are typically set in international standards (like EN 50160) or in national regulations (EIFS 2011:2 for Sweden).

Current emission limits for individual installations are in turn set to prevent such excessive amounts of voltage distortion. Emission limits for individual installations can either be set on a case-by-case basis or by using general limits. The former is the case in most European countries, where planning levels on harmonic emission, together with the source impedance as a function of frequency at the point of connection, are used to obtain location-specific emission limits. Whether the emission from an installation is acceptable or not depends strongly on the specific properties of the grid at that location. A case study is needed for each new installation.

A different approach is used in France [2], where the emission limits are dependent on the short-circuit capacity at the connection point. The resulting limits are still location dependent, but no study is needed to obtain the source impedance as a function of frequency. Only the source impedance at the power-system frequency is needed.

The emission limits as recommended by IEEE Std. 519 [3] are location independent. Instead for each harmonic order, the emission limit is set as a percentage of the maximum current.

\section{E. Comparing with limits}

The measured emission has been compared with the limits according to IEEE Std.519 and the limits as used in France. IEEE Std 519 was introduced to provide direction on dealing with harmonics introduced by static power converters and other nonlinear loads so that power quality problems could be averted. The comparison with IEEE Std.519 is shown in Fig. 4. The figure shows the ratio between the measured emission level (as in Fig. 2) and the emission limit for voltage levels through $69 \mathrm{kV}$. A value above $100 \%$ means that the emission exceeds the limit. It has been assumed that the harmonic spectrum in a $60-\mathrm{Hz}$ system would be the same as in a $50-\mathrm{Hz}$ system.

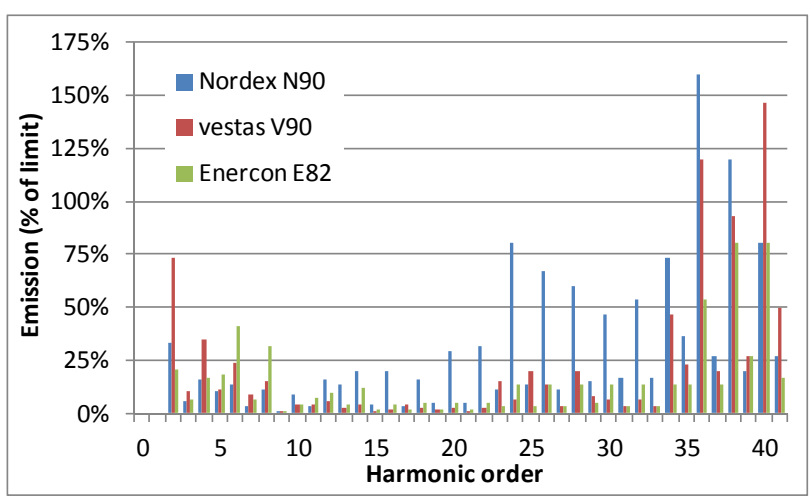

Fig. 4. Emission spectra of the wind turbines as a percentage of the IEEE 519 limits.

The figure shows that the limits are exceeded for harmonics 36, 38 and 40, for the Nordex and Vestas turbines. The emission for all harmonics in this range is relatively high, but the emission limits for even harmonics are only $25 \%$ of the limits for odd harmonics. The requirements are tougher for even harmonics, as these harmonics were not present in the grid at the time when the compatibility levels were set. When the limits in IEEE Std. 519 are strictly followed it will not be allowed to connect these turbines to the grid without additional filtering.

The emission from the three turbines is compared with the French emission limits at medium voltage in Fig. 5. Like the previous figure, this one shows the ratio of the measured emission and the emission limit. None of the harmonics exceeds $50 \%$ of the limit. This means that these turbines can be connected to the French mediumvoltage network without any additional measures.

The French regulation only considers frequencies up to order 25, whereas IEEE Std.519 considers frequencies up to order 41.

A discussion should be started on what are acceptable levels of voltage distortion for even harmonic and for interharmonics. Also the highest order of harmonics considered should be a subject of discussion. The need for filtering is, in the example shown here, determined by the even harmonics. Limits for these have been set long time ago based on equipment available in those days. 


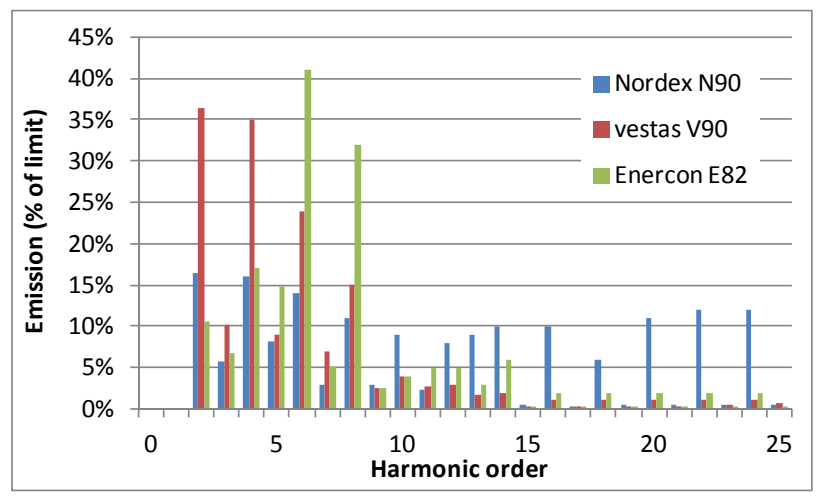

Fig. 5. Emission spectra of the wind turbines as a percentage of the limits for French MV networks.

\section{Harmonic emissions from a wind park}

\section{A. Propagation through a wind park}

With wind farms, the impact of turbines on the grid is not determined by the emission from the individual turbines but by the emission from the park as a whole. The relation between the emissions from individual turbines and from the park as a whole is discussed in [4]. There it is concluded that the emission from a park can be higher as well as lower than the emission that would be obtained by adding the harmonic magnitudes of the individual turbine emissions. Two phenomena contribute to this:

$\checkmark$ The aggregation of the emission from individual sources. The $95 \%$ value of the total emission from a number of individual sources is in most cases less than the sum of the $95 \%$ values. Guidelines for the aggregation from different harmonic sources are given in IEC 61000-3-6, but it is unclear if these rules hold for wind turbines.

$\checkmark$ Due to resonances and damping in the collection grid, the emission from the park can be amplified or damped compared with the aggregation.

Simulations have been performed on a simplified model of a 3-turbine, 10-turbine and 100-turbine park to estimate the combined impact of these two phenomena. For the 3turbine park, the amplification reaches its maximum between 6 and $9 \mathrm{kHz}$ and the emission from the park was, in that frequency range, up to 5 times the emission from one individual turbine, expressed in percent of the rated current. When expressed in Ampere, the emission of the park is 15 times the emission of the individual turbine, but as the rated current of the park equals three times the rated current of one turbine, the total emission is amplified up to five times as a percentage of rated.

\section{B. Emission from a wind park}

Studies of the emission from a wind park as a whole are even more complicated then could be concluded from the earlier discussions. The emission is in this case defined as a distorted current at the point of connection between the public grid and the park. A distinction should thereby be made between "primary emission" and "secondary emission". This distinction is made based on what causes the currents to flow.
Primary emission is caused by the distorted current coming from sources inside of the wind park. This are in most cases the wind turbines, but also powerelectronic devices to control reactive power. Even the turbine and grid transformers are sources of distortion, albeit minor ones in most cases. This primary emission is the one that is normally considered first and often it is the only one considered, thereby neglecting the secondary emission. The secondary emission is due to all other sources of harmonics, outside of the wind park. It is the distorted part of the current that would flow in case there would be no sources of harmonics inside of the wind park.

In this study, only the turbines have been considered. The primary emission from the park is in that case due to the emission from the turbines. But it is not simply the sum of the emissions from the individual turbines. To calculate the primary emission from the park, two phenomena have to be considered: cancellation of harmonic currents from individual turbines; and the propagation from the turbines to the grid. Both phenomena have been considered in a general model of a wind park and applied to 3-turbine, 10- turbine and 100-turbine parks.

The results for a 10-turbine park are shown in Figure 6, where a distinction is made between "individual transfer function" and "overall transfer function”. The individual transfer functions are presented at top in Figure 6, with different colours for different turbine locations, while at bottom the overall transfer function for the 10-turbine wind park is presented.

The individual transfer function gives the relation between a frequency component emitted by an individual turbine and the same frequency component at the point of connection to the grid. All other emission sources are neglected in this case. The individual transfer function is strongly frequency dependent and somewhat different for each turbine location. For this example, the highest value occurs somewhere around $2 \mathrm{kHz}$, where the emission into the grid is almost 20 times the emission from the turbine: this amplification is due to resonances in the collection grid. Studies on secondary emission from wind parks are rare, as the grid code normally does not require this. A study of secondary emission with an off-shore wind park is presented in [5]. The conclusion from that study was that the primary emission is small but that the secondary emission can be several orders of magnitude bigger than the primary emission. The secondary emission is not a concern to the network operator; in fact it has a reducing effect on the voltage distortion from other sources. The secondary emission makes it however difficult to verify if an installation complies with the connection requirements. Secondary emission may also result in high harmonic voltage distortion in the collection grid. 

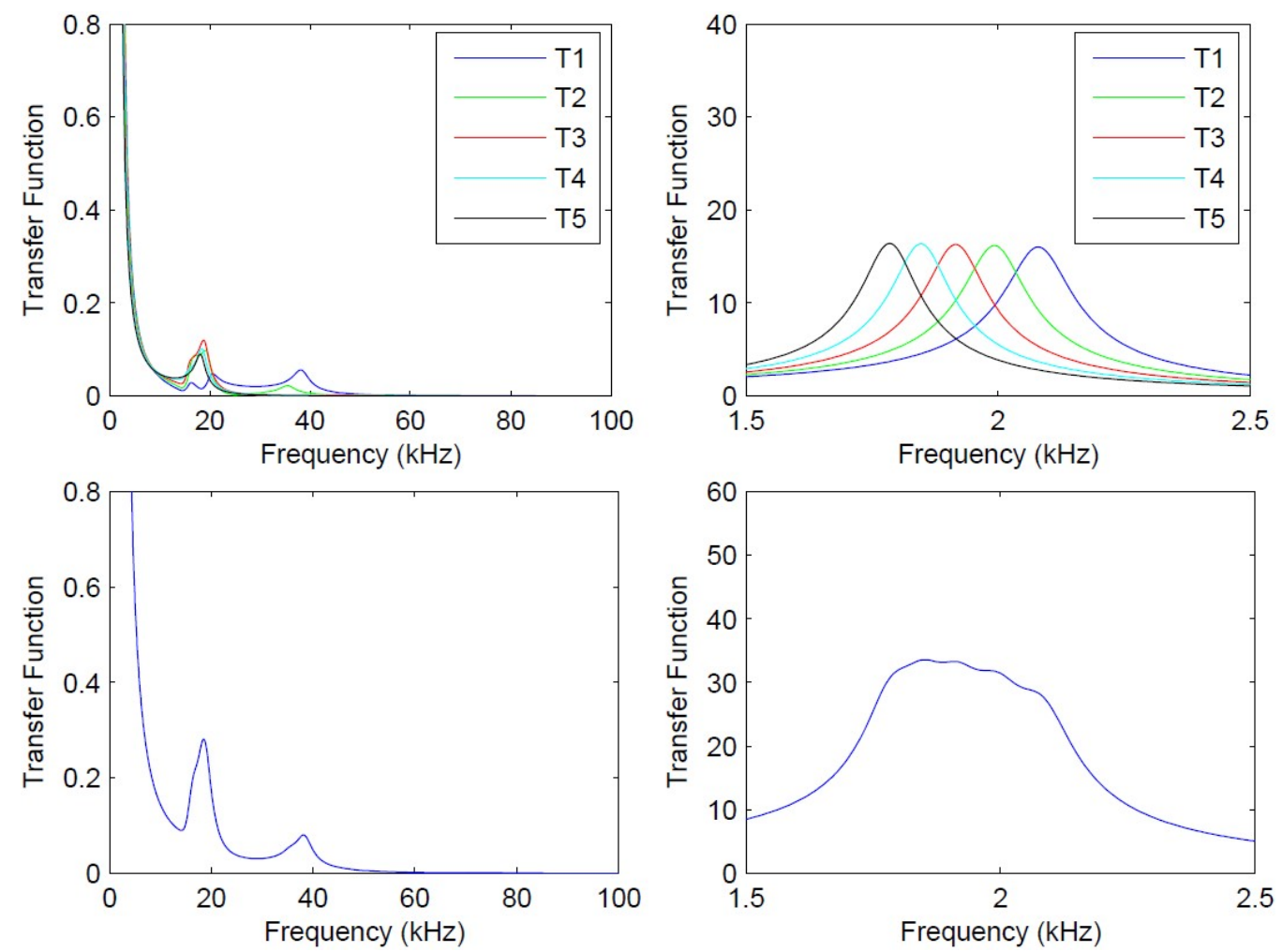

Fig. 6. Individual and overall transfer functions for a 10-turbine wind park.

\section{Conclusion}

Measurements have been performed on the emission from individual wind turbines equipped with power electronics and from a wind park as a whole. The harmonic spectrum of the individual turbines show predominant emission up to order of 10 and increased emission for harmonic range between 35 and 40 . The interharmonic spectrum of the same turbines is similar in magnitude to the emission at harmonic frequencies.

The estimated emission from the wind turbines have been compared with emission limits. The French regulations and IEEE Std.519 have been used as a reference. It is concluded that the emission from individual turbines is below the emission limits in almost all cases. Only higherorder even harmonics exceed the IEEE limits.

Studies were also done on the emission of wind farms as a whole. The distortion of the current at the connection point with the grid was shown not to be a simple addition of the distortion from the individual turbines.

For small wind parks the situation is probably even somewhat better because of aggregation effects between the individual turbines. For large wind parks amplification due to resonances should be considered. Such resonances could occur at frequencies as low as $1 \mathrm{kHz}$. Transmission cables could even further reduce this resonance frequency. This amplification could result in the need for additional filtering when connecting such parks to relatively-weak parts of the grid.

A general conclusion is that harmonic studies are needed before the connection of wind parks, but that these studies should be broader than only comparing the primary emission with the voltage or current limits.

\section{References}

[1] K. Yang, M. Bollen, M. Wahlberg, Characteristic and non-characteristic harmonics from windparks, Int. Conf. Electricity Distribution (CIRED), Frankfurt, June 2011.

[2] Benchmarking report on quality of electricity supply, CEER and ECRB, December 2011.

[3] IEEE Std 519-1992, IEEE Recommended Practices and Requirements for Harmonic Control in Electrical Power Systems, IEEE Std 519-1992

[4] K. Yang, M.H.J. Bollen, L.Z. Yao, Theoretical emission study of wind park grids - emission propagation between windpark and grid, $11^{\text {th }}$ Int Conf on Electric Power Quality and Utilization (EPQU), Lisbon, October 2011.

[5] Y. Chen, M. Bollen, M. Martins, Application of transfer function based harmonic study method to an offshore wind farm, Wind Integration Workshop, Lisbon, November 2012. 